\title{
El desempleo juvenil: un estudio comparado Brasil España
}

\section{Youth unemployment: a comparative study between Brazil and Spain}

\author{
Alicia Garrido Luque ${ }^{1}$ \\ Sabrina Cavalcanti Barros ${ }^{2}$ \\ Sonia Maria Guedes Gondim ${ }^{3}$
}

\begin{abstract}
RESUMEN: En esta investigación se realizó un estudio comparado entre una muestra de jóvenes brasileños y españoles en las que se analizó la relación entre estatus de empleo (empleados versus desempleados) y bienestar psicológico. Se constata una asociación entre desempleo juvenil y deterioro en la salud mental, tanto en la muestra española como en la muestra brasileña. Las diferencias observadas son mayores en el caso de los jóvenes desempleados que en el caso de las jóvenes desempleadas, quienes parecen beneficiarse menos, desde el punto de vista del bienestar psicológico del hecho de tener un empleo. Estas diferencias podrían deberse a la sobrecarga de roles que experimentan las jóvenes, si bien este es un factor que debe ser analizado en futuras investigaciones.
\end{abstract}

Palabras claves: desempleo juvenil; bienestar psicológico; salud mental; estudio transnacional.

ABSTRACT: In this research, a comparative study was conducted among a sample of young Brazilians and Spaniards in which the relationship between employment status (employed versus unemployed) and psychological well-being was analyzed. There is an association between youth unemployment and deterioration in mental health, both in the Spanish sample and in the Brazilian sample. The differences observed are greater in the case of unemployed young men than in the case of unemployed young women, who seem to benefit less, from the point of view of the psychological well-being, of having a job. These differences could be due to the overload of roles that young women experience, although this is a factor that should be analyzed in future research.

Keywords: youth unemployment; psychological well-being; mental health; transnational study.

Las primeras investigaciones sobre los efectos psicosociales del desempleo se centraron principalmente en la población adulta, siendo escasos los estudios en los que se analizó el impacto del desempleo en los jóvenes. Sin embargo, en la actualidad, el desempleo juvenil es considerado un problema grave, dado que este sector es el que presenta los índices más altos de desempleo en la inmensa mayoría de los países industrializados. Así lo dice el último informe de la Organización Internacional del Trabajo [OIT] (2015), donde se destaca que 74 millones de jóvenes en el mundo buscan un empleo, siendo su tasa de desempleo tres veces mayor que la de los adultos. En España, la tasa de desempleo es del 50\% para los jóvenes de entre 16 y 19 años, del 33\% para los que tienen entre 20 y 24 , y de un $22 \%$ para los jóvenes de entre 25 y 29 años (INE, 2017). En Brasil, la crisis económica y política a la que se viene enfrentando el país en los últimos años ha dado lugar a un aumento de la tasa de desempleo entre los jóvenes, que según datos del

\footnotetext{
${ }^{1}$ Doctora en Psicología Social por la Universidad Complutense de Madrid (UCM). Profesora Titular del Departamento de Psicología Social de la UCM - Madrid, España. E-mail: algarrid@cps.ucm.es.

2 Doctora en Psicología por la Universidade Federal de Minas Gerais (UFMG) y por la Universidad Complutense de Madrid (UCM). Profesora del Centro Universitário Unihorizontes - Belo Horizonte, MG, Brasil.

3 Doctora en Psicología por la Universidade Federal do Rio de Janeiro (UFRJ). Profesora del Instituto de Psicología y del Programa de Posgrado en Psicología de la Universidade Federal da Bahia - Salvador, BA, Brasil.
} 
Departamento Intersindical de Estatísticas e Estudos Socioeconômicos [DIEESE] (2017), varía del $38 \%$ en Sao Paulo a un $47 \%$ en el área metropolitana de Salvador.

Estos datos han conducido a que en la actualidad se le conceda mayor importancia a las dimensiones psicológicas y sociales del desempleo juvenil, aunque ya en 1938, Eisenberg y Lazarsfeld señalaban que el desempleo puede afectar a los jóvenes en distintas formas, tales como la imposibilidad de independencia, el deterioro de las relaciones paterno filiales, la disminución de la motivación para el logro, sentimientos de desvalimiento, prolongación del períodos de estudio, aceptación de trabajos precarios, etc.

\section{Efectos psicosociales del desempleo juvenil}

Una de las investigaciones pioneras y clásicas sobre los efectos psicosociales del desempleo es el estudio sociográfico realizado por Jahoda, Lazarsfeld y Zeisel (1933) en la ciudad austriaca de Marienthal, en donde se experimentó un desempleo masivo a causa del cierre de la fábrica textil que proporcionaba trabajo a prácticamente la totalidad de esa comunidad. Este estudio no sólo destaca por ser pionero en el estudio de este tema, sino también por ser una de las pocas investigaciones que ha centrado su análisis en el desempleo como un fenómeno colectivo, dado que con posterioridad la investigación vendría a centrarse en las consecuencias individuales de este fenómeno. Años después, la investigación bibliográfica realizada por Eisenberg y Lazarsfeld (1938), en la que se recogen más de cien referencias de estudios realizados en diferentes países, constituye una muestra de la contribución de las ciencias sociales a la comprensión de los efectos del desempleo. Sus conclusiones subrayan las negativas consecuencias del desempleo para la salud mental: mayor apatía, disminución de la autoestima y un aumento de síntomas de ansiedad y depresivos, entre otros.

Tras unos años en los que el interés por el desempleo pareció disminuir, en las últimas décadas ha vuelto a producirse un aumento de las publicaciones que abordan el análisis de este problema desde una perspectiva psicosocial (e.g., Álvaro \& Garrido, 2002, 2003, 2005; Álvaro, Gondim, Garrido, Luna, \& Dessen, 2012; Gallardo, 2008; Garrido, 1996; Mylonas et al. 2014; Sharma, 2012; Strandh, Winefield, Nilsson, \& Hammarström, 2014).

Siguiendo el enfoque adoptado por autores como Jahoda (1987), el trabajo, entendido como empleo, cumple una serie de funciones que van más allá de la consecución de un salario, su función manifiesta. Podemos afirmar que el trabajo-empleo sirve para dar una imagen de nosotros mismos, al mismo tiempo que provee de una posición y estatus reconocidos socialmente. Asimismo, como señala Jahoda, el trabajo proporciona una estructura temporal, relaciones interpersonales y una actividad. A estas funciones del empleo se las reconoce como funciones expresivas o funciones latentes de las cuales se deriva el significado psicológico y social del trabajo-empleo. Estos aspectos, tomados en conjunto, llevan a destacar la naturaleza social del trabajo, por cuanto es en el proceso de interacción que ocurre en el trabajo donde las personas reciben estímulos que inciden en la construcción de su propia identidad. Otro de los aspectos de los efectos que el trabajo tiene en la identidad de las personas está relacionado con el estatus social dentro de la sociedad. Como indica Jahoda, la opinión pública define el estatus y el prestigio basándose en la naturaleza del puesto de trabajo que tenga una persona. 
En esta línea, autores como Agulló (1997) señalan que no solamente el desempleo puede ser negativo y disfuncional para la persona sino que también el empleo puede serlo, si no posibilita la integración y el desarrollo psicosocial del individuo.

\section{Desempleo Juvenil y bienestar psicológico}

De todos los aspectos que abarca el impacto psicosocial del desempleo el que mayor interés ha suscitado ha sido el que se refiere a sus efectos sobre el bienestar psicológico (Álvaro \& Garrrido, 2002, 2003; Dooley \& Prause, 1995; Feather \& Bond, 1983; Feather \& O'Brien, 1986; Hendry \& Raymond, 1986; Petersen \& Mortimer, 1994; Schaufeli \& Van Yperen, 1992; Sharma, 2012; Strandh et al., 2014). No obstante, algunos autores han señalado que por diversas razones, los jóvenes acusarían menos los efectos psicológicos del desempleo en comparación a la población adulta (Álvaro, 1989; Álvaro \& Garrido, 2003; Furnham, 1994; O`Brien, 1986; Warr, 1987), ya sea por el hecho de carecer de responsabilidades familiares, por la dependencia económica que aún tienen de las figuras paternas y por sus menores compromisos financieros. No obstante, a pesar de las diferencias que puedan presentarse en el bienestar psicológico de desempleados jóvenes y adultos, los primeros pagan un precio muy alto en el desarrollo de su autonomía y competencia por encontrarse en una etapa en la que el trabajo cobra un significado especial.

En general, los estudios que han evaluado los efectos del desempleo juvenil en la salud mental han constatado repetidamente sus negativas consecuencias psicosociales. Por ejemplo, cuando se han comparado muestras de jóvenes empleados y desempleados, se ha observado que estos últimos tienen un menor nivel de bienestar psicológico general (Álvaro, 1989; Álvaro \& Garrido, 2002, 2003; Banks \& Jackson, 1982; Bell \& Blanchflower, 2011; Sharma, 2012; Strandh et al., 2014; Ullah, 1990), experimentan con mayor frecuencia sentimientos de carácter depresivo (Blanch, 1996; Feather \& Bond, 1983) y síntomas de ansiedad (Ullah, Banks, \& Warr, 1985), así como una imagen más negativa de sí mismos y un menor nivel de autoestima (Pettersson, 2012). Igualmente, autores como Fraccaroli, Le Blanc y Hajar (1994) o Meeus, Dekovic y ledema (1997) afirman que la falta de empleo se traduce en un sentimiento de privación relativa y de desvalorización del sí mismo. Como indica Gallardo (2008) el mundo laboral constituye un ámbito de relaciones interpersonales indispensable y necesario para la formación y posterior construcción de la identidad juvenil. El ejercicio del rol laboral se convierte en un factor clave para la adquisición de nuevos rolesidentidades que han de conformar su sí mismo. Como señala Erikson (1968, p.27), "en todo período histórico existen personas que pueden combinar las técnicas dominantes con el desarrollo de su identidad, y que devienen aquello que hacen". Así, el desempleo representa una quiebra vital para los jóvenes, que se sienten incapaces y desvalorizados ante la sociedad y ante sí mismos, pues carecen de un espacio para desarrollarse y prolongar su experiencia biográfica como seres humanos, pues al carecer de un trabajo que para ellos es instrumento de expresión y vinculación personal y social, su formación identitária y su bienestar psicológico se ven afectados negativamente. Podemos por tanto, afirmar que el desempleo representa para los jóvenes una experiencia psicológicamente desestabilizadora.

El marco anteriormente descrito nos lleva a presentar la siguiente investigación cuyo objetivo fue mostrar la relación entre desempleo juvenil y bienestar psicológico, en dos contextos diferentes como son España y Brasil. 


\section{Método}

\section{Participantes y Procedimiento}

En este estudio correlacional se investiga la relación entre desempleo y bienestar psicológico. Para ello se seleccionó una muestra no probabilística de jóvenes empleados y desempleados menores de 30 años $(N=212)$.

En Brasil, participaron 115 jóvenes, de los cuales el $66.7 \%$ eran mujeres y el $33.3 \%$ hombres. El 44.3\% ( $n=51)$ estaban empleados y el 63.7\% $(n=64)$ estaban desempleados. En España, participaron 97 jóvenes de los cuales el 70\% eran mujeres y el 30\% restante hombres. El 35\% $(n=28)$ estaban empleados y el $65 \%(n=63)$ desempleados. En Brasil, los desempleados respondieron a un cuestionario en los servicios de SINEBAHIA (Servicio Público de Intermediación para el Trabajo). Esta institución se dedica a labores de selección y entrenamiento de personas que buscan un puesto de trabajo. En el caso español, los participantes lo hicieron en las oficinas del INEM (Instituto Nacional de Empleo). En cuanto a los trabajadores empleados, en el caso de Brasil fueron reclutados en cursos de capacitación pública y privada, mientras que en el caso español fueron seleccionados en empresas privadas e instituciones estatales. En ambos casos los cuestionarios fueron administrados por personal entrenado. Los entrevistados respondieron a un cuestionario en el que se incluyó una media de salud mental como es el General Health Questionnaire (GHQ) de Goldberg $(1972,1978)$, formada por 12 ítems. Se trata de un cuestionario autoadministrable, en un principio diseñado para detectar trastornos psíquicos menores, de carácter no psicótico. El coeficiente alfa de consistencia interna fue de 0.89 en el caso español y de 0.86 para la muestra brasileña.

\section{Resultados}

Para comprobar la existencia o no de una asociación entre desempleo y deterioro psicológico se realizó un ANOVA factorial por el procedimiento univariante con el que analizamos si el bienestar psicológico de los jóvenes empleados y desempleados difería significativamente. Los resultados obtenidos permiten confirmar las diferencias entre empleados $(M=10.58 ; D T=6.10)$ y desempleados $(M=14.40 ; D T=6.18), F_{(1,211)}=13.78, p$ $<.001$. No se observaron efectos significativos del país en el bienestar psicológico $F_{(1,211)}=$ $2.51, n s$, ni una interacción significativa entre el país y el estatus ocupacional $F_{(1,211)}=1.14$, $n s$. Tal y como podemos ver en las figuras 1 y 2 , existe una asociación significativa entre desempleo y deterioro psicológico en ambos países. 


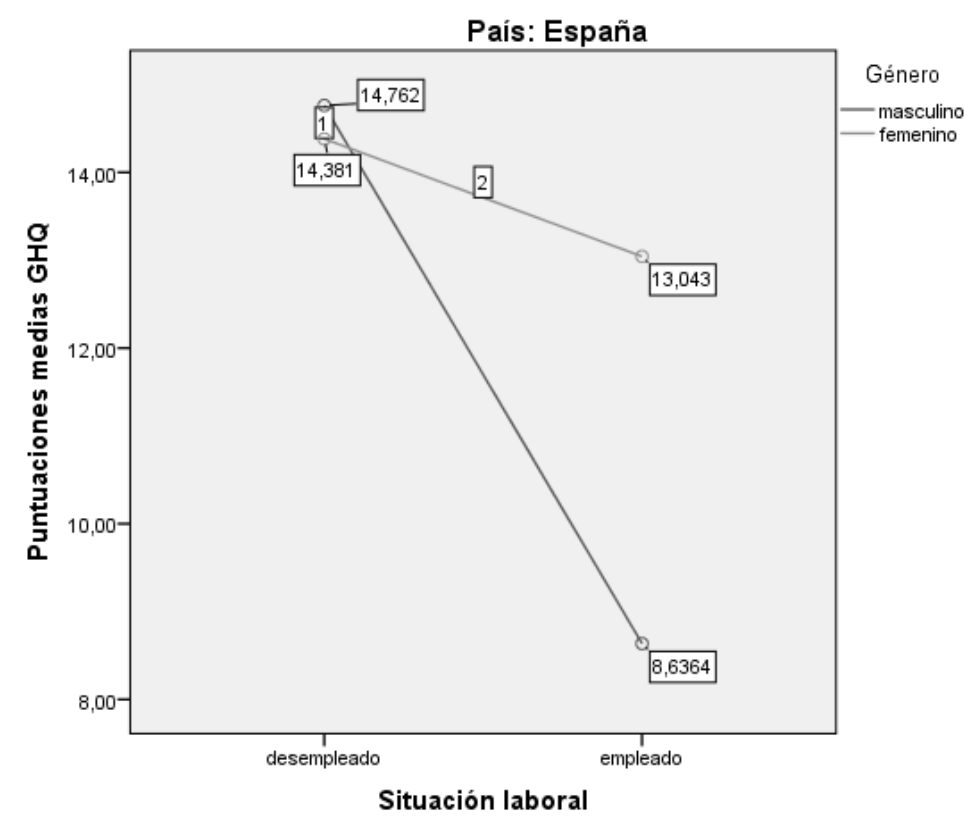

Figura 1 - Situación laboral de los jóvenes y bienestar psicológico en España

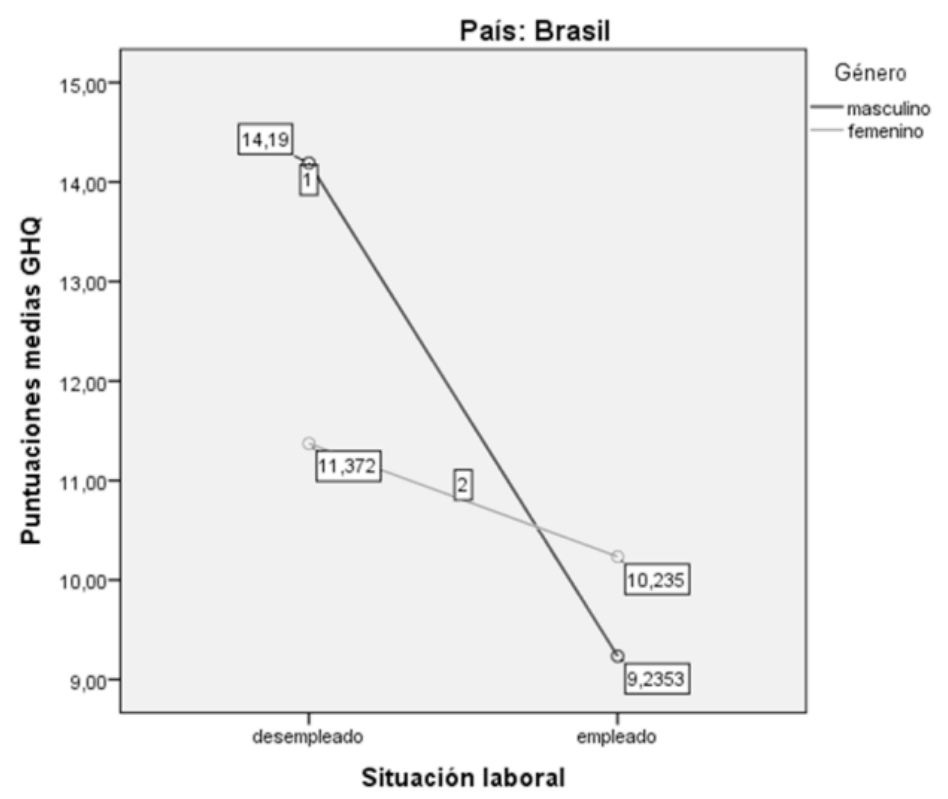

Figura 2 - Situación laboral de los jóvenes y bienestar psicológico en Brasil

Asimismo, se constató una interacción significativa entre el estatus ocupacional y el sexo, $F_{(1,211)}=5.55, p<.01$. Tanto en Brasil como en España, el desempleo tuvo un impacto negativo en la salud mental tanto en el caso de los hombres como en el de las mujeres. Sin embargo, el hecho de tener un empleo tiene una influencia más positiva para los hombres. Las diferencias en salud mental entre empleados y desempleados fueron mucho más pronunciadas en el caso de los varones que en el de las mujeres, cuya salud mental no parece beneficiarse tanto del hecho de tener un empleo. La interacción estatus ocupacional $\mathrm{x}$ sexo $\mathrm{x}$ país no resultó significativa, $F(1,211)=0.71, n \mathrm{~s}$. 


\section{Conclusiones}

Los resultados de este estudio constatan la existencia de una asociación entre el desempleo juvenil y el bienestar psicológico. A pesar de que hay algunos factores que podrían hacer que el impacto del desempleo fuera menor en el caso de los jóvenes, como las menores responsabilidades económicas y familiares que estos tienen, los datos presentados sugieren que el deterioro psicológico de las personas desempleadas no depende únicamente de la mayor o menor necesidad económica de trabajar, sino también de las funciones psicológicas que cumple el empleo. La experiencia del desempleo en las primeras fases de la carrera laboral puede convertirse en un obstáculo para la integración en la sociedad adulta y el desarrollo de una identidad profesional. En este sentido, los datos de este estudio corroboran la hipótesis de que el desempleo tiene un impacto negativo en la salud mental también para los jóvenes (Álvaro \& Garrido, 2002, 2003; Bell \& Blanchflower, 2011; Sharma, 2012; Strandh et al., 2014; Ullah, 1990; etc.).

Otra conclusión que se desprende de los resultados de este estudio es que los efectos del estatus de empleo en la salud mental son diferentes para hombres que para mujeres. Aunque en ambos casos se observa un mayor deterioro psicológico entre las personas desempleadas, las diferencias no son tan acusadas en el caso de las mujeres. Los menores beneficios psicológicos que el empleo parece tener en el caso de las mujeres podrían deberse a los efectos de la sobrecarga de roles. Si bien los resultados aquí obtenidos no permiten confirmar esta hipótesis, existe evidencia proveniente de diferentes meta-análisis sobre las negativas consecuencias que implica el conflicto de roles entre las demandas del trabajo y la vida familiar, especialmente para la mujeres, afectando su bienestar psicológico (Byron, 2005; Ford, Heinen \& Langkamer, 2007). Si bien España y Brasil son dos países con tasas de desempleo juvenil muy diferentes, mercados laborales desiguales y unas tasas de cobertura por desempleo muy diferenciadas, se constata que el desempleo juvenil está asociado a menores niveles de bienestar psicológico. Si bien las muestras aquí obtenidas no tienen un carácter representativo, apuntan a un patrón similar, lo que contribuye a confirmar los resultados obtenidos en otros estudios y recogidos en la ya extensa bibliografía sobre las negativas consecuencias psicosociales del desempleo para los jóvenes a la que nos hemos referido con anterioridad.

\section{Referencias}

Agulló, E. (1997). Jóvenes, trabajo y identidad. Oviedo: Universidad de Oviedo.

Álvaro, J. L. (1989). Desempleo juvenil y salud mental. In J. R. Torregrosa, J. Bergere, \& J. L. Álvaro (Eds). Juventud, trabajo y desempleo: un análisis psicosociológico (pp. 305-319). Madrid: Ministerio del Trabajo.

Álvaro, J. L., \& Garrido, A. (2002). Reflejos da situaçao laboral na saúde mental dos jovens. In A. Mendes, L. O. Borges, \& M. C. Ferreira (Eds). Trabalho em transição, saúde em risco (pp. 207-224). Brasilia: Finatec.

Álvaro, J. L., \& Garrido, A. (2003). Economic hardship, unemployment status and psychological well-being. In T. Hammer (Ed). Youth unemployment and social exclusión in Europe (pp. 173-192). Bristol: Policy Press.

Álvaro, J. L., \& Garrido, A. (2005). Youth unemployment and job-seeking behaviour in Europe. In H. Bradley \& J. Van Hoof (Eds). Young people in Europe. Labour markets and citizenship (pp. 81-98). Bristol: Policy Press.

Álvaro, J. L., Gondim, S., Garrido, A., Luna, A. F., \& Dessen, M. C. (2012). Desempleo y bienestar psicológico en Brasil y España: Estudio comparativo. Revista Psicologia: Organizações e Trabalho, 12(1), 5-16. 
Banks, M. H., \& Jackson, P. R. (1982). Unemployment and the risk of minor Psychiatric disorder in young people: Cross-sectional and longitudinal evidence. Psychological Medicine, 12(4), 789-798.

Bell, D., \& Blanchflower, D. (2011). Young people and the great recession. Oxford Review of Economic Policy, 27(2), 241-267.

Blanch, J. M. (1996). Psicología Social del Trabajo. In J. L. Álvaro, A. Garrido, \& J. R. Torregrosa (Eds). Psicología Social Aplicada (pp. 85-119). Madrid: Mc Graw-Hill.

Byron, K. (2005). A meta-analytic review of work-family interference and its antecedents. Journal of Vocational Behavior, 67(2), 169-198. doi: 10.1016/j.jvb.2004.08.009.

Departamento Intersindical de Estatística e Estudos Socioeconômicos (2017). País segue mergulhado em crise política econômica. $\quad$ Recuperado de https://www.dieese.org.br/boletimdeconjuntura/2017/boletimConjuntura011.html.

Dooley, D., \& Prause, J. (1995). Effects of unemployment on school leavers self-esteem. Journal of Occupational and Organizational Psychology, 68(3), 177-192. doi: 10.1111/j.2044-8325.1995.tb00580.x.

Eisenberg, P., \& Lazarsfeld, P. F. (1938). The psychological effects of unemployment. Psychological Bulletin, 35(6), 358-390. doi: http://dx.doi.org/10.1037/h0063426.

Erikson, E. H (1968). Identidad, juventud y crisis. Buenos Aires: Paidós.

Feather, N. T., \& Bond, M. J. (1983). Time structure and purposeful activity among employed and unemployed university graduates. Journal of Occupational Psychology, 56(3), 241-254. doi: 10.1111/j.20448325.1983.tb00131.x.

Feather, N. T., \& O'brien, G. (1986). A longitudinal study of the effects of employment and unemployment on school-leavers. Journal of Occupational Psychology, 59(2), 121-144. doi: 10.1111/j.20448325.1986.tb00219.x.

Ford, M. T., Heinen, B. A., \& Langkamer, K. L. (2007). Work and family satisfaction and conflict: A meta-analysis of cross-domain relations. Journal of Applied Psychology, 92(1), 57-80. doi: 10.1037/0021-9010.92.1.57.

Fraccaroli, F., Le-Blanc, A., \& Hajjar, V. (1994). Social self-description and affective well-being in young unemployed people: a comparative study. European Work and Organizational Psychologist, 4(2), 81100. doi: http://dx.doi.org/10.1080/13594329408410477.

Furnham, A. (1994). The psychosocial consequences of youth unemployment. In Petersen, A. C, \& Mortimer, O. T (Eds). Youth Unemployment and Society (pp. 199-226). Cambridge: Cambridge University Press.

Gallardo, J. (2008). Juventud, trabajo, desempleo e identidad: un enfoque psicosocial. Madrid: Universidad Complutense de Madrid.

Garrido, A. (1996). Psicología social del desempleo. In J. L. Álvaro, A. Garrido, \& J. R. Torregrosa (Eds). Psicología Social Aplicada (pp. 121-154). Madrid: McGraw-Hill.

Goldberg, D. P. (1972). The detection of psychiatric illness by questionnaire. Londres: Oxford University Press.

Goldberg, D P. (1978). Manual for the General Health Questionnaire. Windsor: National Foundation for Educational Research.

Hendry, L. B., \& Raymond, M. J. (1986). Psycho-social aspects of youth unemployment: an interpretative theoretical model. Journal Adolescence, 9(4), 355-366. doi: 10.1016/S0140-1971(86)80041-0.

Instituto Nacional de Estadística (2017). Encuesta de Población Activa. Recuperado de http://www.ine.es/dyngs/INEbase/es/operacion.htm?c=Estadistica_C\&cid=1254736176918\&menu=ulti Datos\&idp $=1254735976595$.

Jahoda, M. (1987). Empleo y desempleo: Un análisis socio-psicológico. Madrid: Morata.

Jahoda, M., Lazarsfel, P.F., \& Zeisel, H. (1933). Los Parados de Marienthal. Madrid: Morata.

Meeus, W., Dekovic, M., \& ledema, J. (1997). Unemployment and identity in adolescente: A social comparison perspective. Career-development Quartely, 45(4), 369-380. doi: 10.1002/j.2161-0045.1997.tb00540.x 
Mylonas, K., Furham, A., Divale, W, Leblebici, C., Gondim, S., Moniz, A., \& Boski, P. (2014). Bias in Terms of Culture and a Method for Reducing It: An Eight-Country 'Explanations of Unemployment Scale' Study. Educational and Psychological Measurement, 74(1), 77-96. doi: 10.1177/0013164413502669.

O’Brien, G. (1986). Psychology of work and unemployment. Nueva York: John Willey.

Organización Internacional del Trabajo (2015). Tendencias Mundiales del Empleo, el informe Perspectivas sociales y del empleo en el mundo - Tendencias 2015. Ginebra: International Labour Office.

Petersen, A. C., \& Mortimer, O. T. (1994). Youth Unemployment and Society. Cambridge: Cambridge University Press.

Pettersson, P. (2012). Does unemployment have any effects on self-esteem and does social support, coping and perceived control have positive impacts on self-esteem during unemployment? Gävle: University of Gävle.

Sharma, A. (2012). Well-being of youth: Impact of unemployment. Advances in Asian Social Science, 2(4), 539544.

Shaufeli, N. W., \& Van Yperen. (1992). Unemployment and psychological distress among graduates a longitudinal study. Journal of Occupational and Organizational Psychology, 65(44), 291-305.

Strandh, M., Winefield, A., Nilsson, K., \& Hammarström, A. (2014). Unemployment and mental health scarring during the life course. The European Journal of Public Health, 24(3), 440-445. doi: 10.1093/eurpub/cku005

Ullah, P. (1990). The association between income, financial strain and psychological well-being among unemployed youth. Journal of Occupational Psychology, 63(4), 317-330. doi: 10.1111/j.20448325.1990.tb00533.x

Ullah, P., Banks, M., y Warr, P. (1985). Social support, social pressures and psychological distress during unemployment. Psychological Medicine, 15(2), 283-295.

Warr, P. B. (1987). Work, unemployment and mental health. Oxford: Oxford University Press. 